\title{
Developing Clostridia as Cell Factories for Short- and Medium-Chain Ester Production
}

\author{
Qingzhuo Wang', Naief H. Al Makishah'2, Qi Li³, Yanan Li', Wenzheng Liu', \\ Xiaoman Sun ${ }^{1 *}$, Zhiqiang Wen ${ }^{1 *}$ and Sheng Yang ${ }^{4,5}$
}

${ }^{1}$ School of Food Science and Pharmaceutical Engineering, Nanjing Normal University, Nanjing, China, ${ }^{2}$ Department of Environmental Sciences, Faculty of Meteorology, Environment and Arid Land Agriculture, King Abdulaziz University, Jeddah, Saudi Arabia, ${ }^{3}$ College of Life Sciences, Sichuan Normal University, Chengdu, China, ${ }^{4}$ Huzhou Center of Industrial Biotechnology, Shanghai Institutes of Biological Sciences, Chinese Academy of Sciences, Shanghai, China, ${ }^{5}$ Key Laboratory of Synthetic Biology, CAS Center for Excellence in Molecular Plant Sciences, Shanghai Institute of Plant Physiology and Ecology, Chinese Academy of Sciences, Shanghai, China

\section{OPEN ACCESS}

Edited by:

Chuang Xue,

Dalian University of Technology, China

Reviewed by:

Adam William Westbrook,

Genecis Bioindustries Inc., Canada

Angel León-Buitimea,

Universidad Autonoma de Nuevo

Leon, Mexico

*Correspondence:

Zhiqiang Wen

zqwen@njnu.edu.cn

Xiaoman Sun

xiaomansun@njnu.edu.cn

Specialty section:

This article was submitted to

Synthetic Biology,

a section of the journal

Frontiers in Bioengineering and

Biotechnology

Received: 31 January 2021

Accepted: 19 April 2021

Published: 07 June 2021

Citation:

Wang Q, Al Makishah NH, Li Q,

Li Y, Liu W, Sun X, Wen Z and Yang S

(2021) Developing Clostridia as Cell

Factories for Short-

and Medium-Chain Ester Production.

Front. Bioeng. Biotechnol. 9:661694.

doi: 10.3389/fbioe.2021.661694
Short- and medium-chain volatile esters with flavors and fruity fragrances, such as ethyl acetate, butyl acetate, and butyl butyrate, are usually value-added in brewing, food, and pharmacy. The esters can be naturally produced by some microorganisms. As ester-forming reactions are increasingly deeply understood, it is possible to produce esters in non-natural but more potential hosts. Clostridia are a group of important industrial microorganisms since they can produce a variety of volatile organic acids and alcohols with high titers, especially butanol and butyric acid through the CoAdependent carbon chain elongation pathway. This implies sufficient supplies of acylCoA, organic acids, and alcohols in cells, which are precursors for ester production. Besides, some Clostridia could utilize lignocellulosic biomass, industrial off-gas, or crude glycerol to produce other branched or straight-chain alcohols and acids. Therefore, Clostridia offer great potential to be engineered to produce short- and medium-chain volatile esters. In the review, the efforts to produce esters from Clostridia via in vitro lipase-mediated catalysis and in vivo alcohol acyltransferase (AAT)-mediated reaction are comprehensively revisited. Besides, the advantageous characteristics of several Clostridia and clostridial consortia for bio-ester production and the driving force of synthetic biology to clostridial chassis development are also discussed. It is believed that synthetic biotechnology should enable the future development of more effective Clostridia for ester production.

Keywords: Clostridium, ester, lipase, alcohol acyltransferase, synthetic biology

\section{INTRODUCTION}

Short- and medium-chain volatile esters $(\mathrm{C} 2-\mathrm{C} 12)$ with flavors and fruity fragrances are usually value-added in brewing, food, and pharmacy (Rodriguez et al., 2014; Aleksander et al., 2019). For example, ethyl acetate, ethyl lactate, butyl acetate, and ethyl hexanoate are the main components of the flavor substances in Baijiu (Chinese liquor) (Yi et al., 2019). With the expansion of the application field, the demand of esters continues to rise in recent years. 
Traditionally, short- and medium-chain fatty acid esters are mainly produced by concentrated sulfuric acid-mediated esterification of acids and alcohols (Cull et al., 2003). This method has certain risks in terms of safety, health, and environment, because it usually causes serious equipment corrosion, as well as a large amount of wastewater and residues (Jermy and Pandurangan, 2005). The recently developed ionic liquid catalytic method can alleviate these problems to some extent, but it is expensive and not stable (Tankov et al., 2017). Compared to chemical methods, biosynthesis via enzyme catalysis or metabolic engineering is much more environmentally friendly and is expected to be an alternative. Indeed, the esters can be naturally produced by some yeasts and lactic acid bacteria, but the efficiency is far from satisfactory (Mukdsi et al., 2009; Kruis et al., 2018a). Therefore, a lot of efforts have been paid to develop nonnatural but more potential strains as microbial cell factories for short- and medium-chain volatile ester production (Rodriguez et al., 2014; Kruis et al., 2017).

Clostridia are especially suitable hosts for ester production due to the diversity of abundant precursors, substrates, and products (Moon et al., 2016; Noh et al., 2019). In the review, we summarized the advances of ester production by Clostridia including in vitro lipase catalysis and in vivo acyltransferase reaction. Besides, we suggested some promising clostridial chassis for bio-ester production and discussed the driving force of synthetic biology in this field.

\section{ENZYMES AND PATHWAYS IN MICROORGANISMS FOR ESTER PRODUCTION}

There are mainly four kinds of ester-forming reactions reported in microorganisms that naturally produce esters. Correspondingly, four kinds of ester synthases including esterase (lipase), hemiacetal dehydrogenase, Baeyer-Villiger monooxygenases, and alcohol acyltransferase (AAT) are involved (Aleksander et al., 2019). Among them, esterase and AATmediated reactions are often used for ester overproduction (Aleksander et al., 2019; Noh et al., 2019).

It is a classic strategy to adapt lipase to catalyze the esterification reaction of organic acids and short-chain alcohols (Stergiou et al., 2013; Aleksander et al., 2019). Unfortunately, for the in vitro catalytic system, enzymes and substrates need to be produced dedicatedly, which caused complicated process routes and huge equipment costs in most cases (Stergiou et al., 2013; Aleksander et al., 2019).

Theoretically, the integration of enzyme and substrate production and catalytic reaction in one reactor is expected to dramatically reduce costs. Therefore, metabolic engineering by the condensation of alcohols and acyl-CoA using AATs is an emerging strategy, which enables some microorganisms, such as Escherichia coli, Saccharomyces cerevisiae, and Kluyveromyces marxianus, to produce bio-esters (Rodriguez et al., 2014; Kruis et al., 2017; Löbs et al., 2017; Bohnenkamp et al., 2020). The reported AATs are mainly derived from yeast, including S. cerevisiae, K. marxianus, Saccharomyces bayanus, and Saccharomyces uvarum (Fujii et al., 1996; Yoshimoto et al., 1998; Saerens et al., 2006). For example, in S. cerevisiae, alcohol acetyltransferases are mainly encoded by the genes ATF1, ATF2, EEB1, EHT1, and EAT1 (Fujii et al., 1996; Lilly et al., 2006; Saerens et al., 2006; Kruis et al., 2018a,b). These genes have a certain compensatory effect on each other, but the catalytic activity and substrate preference of these AATs are not completely the same. Besides, AATs from different strain sources usually exhibited different catalytic activities and substrate selectivity, which explains why E. coli produces multiple esters after different (and even the same) AATs are introduced (Rodriguez et al., 2014).

In addition to the thoughtful selection and refined expression of AATs, challenges to balance the metabolic pathways for rational precursor distribution need to be addressed. This is because acyl-CoA is an indispensable precursor of esters, as well as fatty acids and alcohols. The metabolism of esters, fatty acids, and fatty alcohols inevitably competes for acyl-CoA (Aleksander et al., 2019; Noh et al., 2019). In E. coli, S. cerevisiae and K. marxianus, acyl-CoA is usually of tight supply, and the metabolic and regulatory networks for acyl-CoA synthesis and consumption are rather complicated. It challenges in distributing precursors reasonably (Rodriguez et al., 2014; Kruis et al., 2017; Löbs et al., 2017).

In contrast, Clostridia can produce a variety of volatile organic acids and alcohols (Figure 1), especially butanol and butyrate through the CoA-dependent carbon chain elongation pathway (Tracy et al., 2012; Cho et al., 2015). This implies sufficient supplies of acyl-CoA, organic acids, and alcohols in cells, which are precursors for ester production. Therefore, Clostridia offer great potential to be engineered to produce shortand medium-chain volatile esters. Recently, many important progresses, including lipase catalysis of fermentation broth, AAT heterologous expression, and exploration of different clostridial chassis, have been made in Clostridia-assisted ester production.

\section{LIPASES MEDIATED ESTERIFICATION OF ALCOHOLS AND ACID FROM CLOSTRIDIA}

Clostridium is a very important category of prokaryotes, composed of nearly 200 different species (Wiegel et al., 2006). Some non-pathogenic species such as Clostridium acetobutylicum and Clostridium beijerinckii are known as solventogenic Clostridia because they can utilize starch, molasses, and other sugars to produce bulk chemicals such as ethanol, butanol, and acetone (Lee et al., 2008). Some other species can directly use lignocellulose, glycerol, or syngas $\left(\mathrm{H}_{2} / \mathrm{CO}_{2}, \mathrm{CO}\right)$ as a sole carbon source to produce short-chain organic acids or alcohols (Ren et al., 2016).

These alcohols and acids are natural substrates for lipasecatalyzed esterification reactions. Therefore, adding lipase to the clostridial fermentation broth is a common strategy for ester production (Noh et al., 2019). In 2013, van den Berg et al. (2013) realized butyl butyrate biosynthesis, by adding commercially available Candida antarctica lipase B (CaLB; Novozym 435) into the fermentation broth of C. acetobutylicum. Meanwhile, 


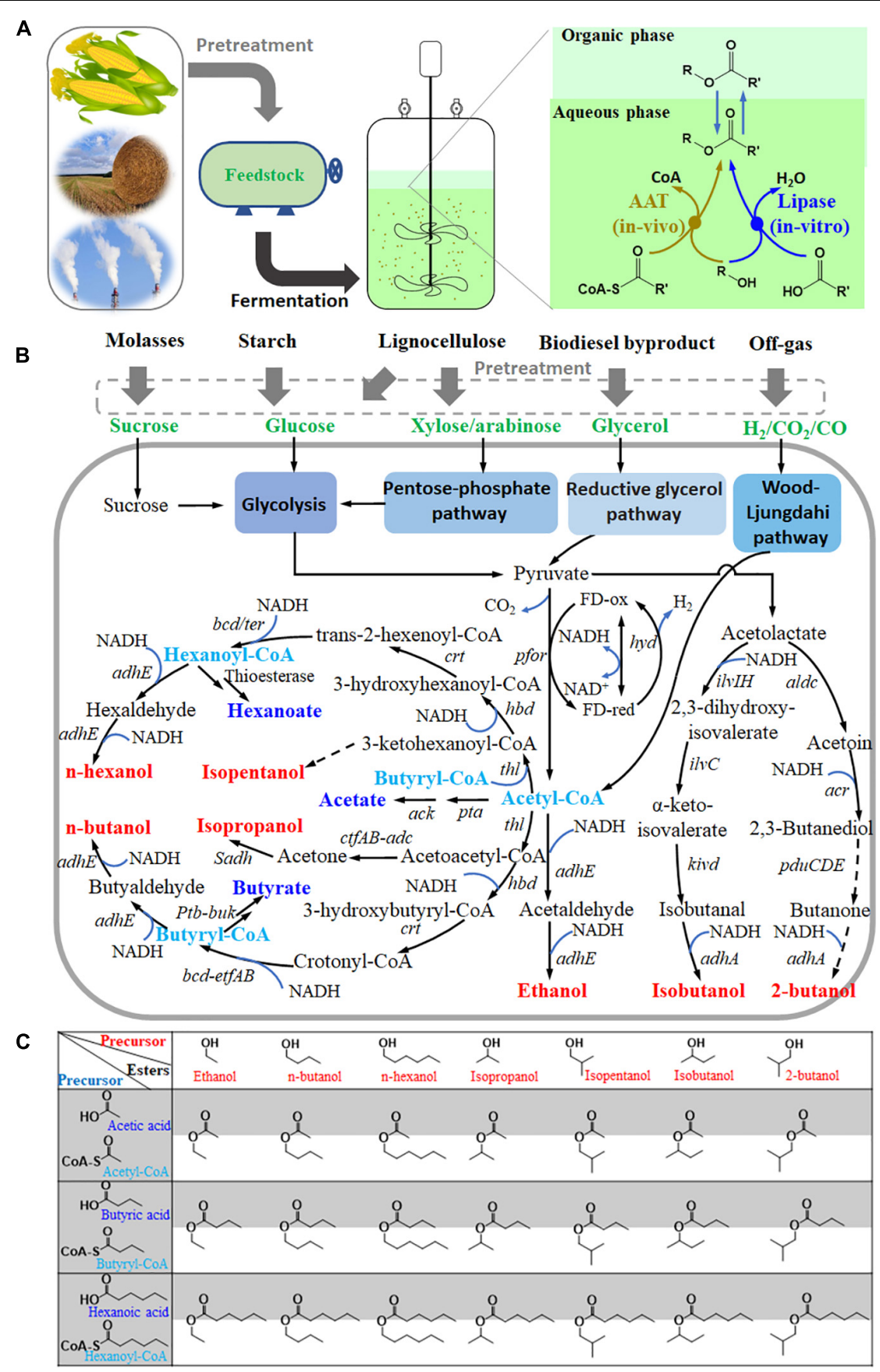

FIGURE 1 | Clostridia are promising cell factories for short- and medium-chain ester production. (A), process and methods for ester production by Clostridia from various feedstocks; (B), metabolic pathway for ester precursors (short-chain acids or acyl CoA, and alcohols) in some Clostridia; (C), matrix of esters putatively produced by Clostridia based on the above precursors. pfor, pyruvate: ferredoxin oxidoreductase; thl, acetyl-CoA acetyltransferase; $h b d$, beta-hydroxybutyryl-CoA dehydrogenase; crt, crotonase; bcd/ter, butyryl-CoA dehydrogenase; adhE or adhE1/2, acetaldehyde/ethanol dehydrogenase; pta, phosphotransacetylase; ack, acetate kinase; ctfAB, acetate/butyrate-acetoacetate COA-transferase; adc, acetoacetate decarboxylase; Sadh, primary/secondary alcohol dehydrogenase; ptb, phosphate butyryl-transferase; buk, butyrate kinase; hyd, ferredoxin hydrogenase; bdhA/B, butanol dehydrogenase; edh, alcohol dehydrogenase; ilvIH, acetolactate synthase; ilvC, keto-acid reductoisomerase; kivd, $\alpha$-ketoisovalerate decarboxylase; adhA, alcohol dehydrogenase; aldc, acetolactate decarboxylase; acr, acetoin reductase; pduCDE, diol dehydratase. Dash lines indicate putative pathways.

hexadecane was adapted to extract butyl butyrate to the organic phase, which helped in product separation and reduced the toxicity of butyl butyrate. Unfortunately, the butyl butyrate titer is only $4.9 \mathrm{~g} / \mathrm{L}$ in the extractant phase because of low butyrate accumulation during ABE fermentation. Therefore, butyrate was supplemented in a later study in Clostridium sp. 
strain BOH3 broth (Xin et al., 2016). As for the fermentation broth of Clostridium tyrobutyricum, a butyrate hyper-producing strain, butanol supplementation is necessary for butyl butyrate production (Zhang et al., 2017).

In order to reduce substrate costs and fermentation process complexity caused by butyrate or butanol addition, a clostridial consortium composed of $C$. tyrobutyricum and $C$. beijerinckii was established (Cui et al., 2020a). In the consortium, butyrate produced by $C$. tyrobutyricum and butanol and isopropanol produced by $C$. beijerinckii are catalyzed by exogenous lipase to form butyl butyrate and isopropyl butyrate, respectively. In another study (Seo et al., 2017), a C. beijerinckii spo0A (a critical regulator to shift metabolism from acidogenesis to solventogenesis) mutant was used for ester production. Since transition from butyrate to butanol is disrupted in the strain, it could produce more butyrate but less butanol compared with the wild type. Accordingly, the addition of butyrate and butanol is avoided, but exogenous lipase is still indispensable.

Although it has been reported that indigenous lipases of Clostridium sp. strain $\mathrm{BOH} 3$ can be induced by olive oil or Bio-OSR (Xin et al., 2016), indigenous lipases (with low expression level and enzymatic activity) are often not enough, and additional lipases are still required to further increase butylbutyrate production. What is more, lipase source, lipase loading dosage, and other factors like extractant, agitation speed, and $\mathrm{pH}$ also affected the performance of lipase-mediated esterification in fermentation broth (Xin et al., 2016; Zhang et al., 2017).

The bottleneck of lipase-mediated esterification lies in the cost of exogenously added lipase. Fortunately, immobilized lipase and optimal reaction conditions may make the cost acceptable. An alternative option is overexpressing and secreting heterologous lipases by Clostridium. However, there has been almost no progress in lipase overexpression in Clostridium until now, because it is really challenging to engineer Clostridium to secrete proteins well (Wen et al., 2020c). By comparison, the in vivo AATs-dependent pathway is more thermodynamically favorable in an aqueous fermentation environment, but more dependent on hosts (Noh et al., 2019).

\section{ALCOHOL ACYLTRANSFERASE-MEDIATED ESTER SYNTHESIS IN CLOSTRIDIA}

Ester production in Clostridia by condensation of alcohols and acyl-CoA can be traced back to 2006 (Horton and Bennett, 2006). Horton and Bennett (2006) successfully overexpressed the ATF2 gene from $S$. cerevisiae in wild $C$. acetobutylicum and a mutant strain M5, respectively, and realized butyl acetate production in strain M5 from glucose for the first time. Noh et al. also observed bio-ester synthesis in C. acetobutylicum after introducing alcohol acyltransferases (AATs) from Fragaria $x$ ananassa (strawberry) or Malus sp. (apple), respectively (Noh et al., 2018). Interestingly, butyl butyrate accounted for about $90 \%$ of the total esters, while butyl acetate accounted for a very small proportion. In two recent studies, Fang et al. (2020) and Li et al. (2020) expressed ATF1 in C. beijerinckii and Clostridium diolis, respectively; the generated strains produced 5.42 and $1.37 \mathrm{~g} / \mathrm{L}$ of butyl acetate as the main ester products from glucose. The type difference in the main ester products in the above studies may be attributed to substrate selectivity of AATs from different sources (Noh et al., 2018; Aleksander et al., 2019).

In addition to mesophilic Clostridia, the thermophilic Clostridia are also promising hosts for bio-ester production, because higher temperature could facilitate the downstream ester separation (Mazzoli and Olson, 2020). Seo et al. (2019) demonstrated that a thermostable chloramphenicol acetyltransferase from Staphylococcus aureus (CATSa) can work as a potential AAT in Clostridium thermocellum. CATSa heterologous expression in $C$. thermocellum has enabled the production of ethyl acetate and isobutyl acetate directly from cellulose. A potential drawback is ester degradation caused by the endogenous carbohydrate esterases (CEs), which hindered ester accumulation in vivo. Therefore, in a subsequent study, Seo et al. identified and disrupted two putative CEs (encoded by Clo1313_0613 and Clo1313_0693) in C. thermocellum, which alleviated ester degradation and further improved isobutyl acetate production by almost 10-fold (Seo et al., 2020).

Generally, Clostridia have been proven to be potential cell factories for ester production. However, these studies mainly focus on the construction and optimization of the ester production process in Clostridia. The characteristics and advantages of Clostridia have not been fully utilized.

\section{SEVERAL POTENTIAL CLOSTRIDIAL HOSTS FOR ESTER SYNTHESIS}

Clostridial hosts for ester synthesis have been expanded from typical solventogenic Clostridia to some unconventional strains. These Clostridia with advantageous characteristics in substrate utilization and ester precursor accumulation are worth exploring and developing as novel ester production chassis.

Clostridium tyrobutyricum is one of the most efficient butyrate-producing Clostridia, which can produce about $50 \mathrm{~g} / \mathrm{L}$ butyrate in batch fermentation under optimized culture conditions (Fu et al., 2017; Bao et al., 2020). Different from the solventogenic Clostridia such as C. acetobutylicum and $C$. beijerinckii, there is a special acetate and butyrate reassimilation mechanism in the strain. With its unique CoA transferase CAT1, acetate and butyrate can be efficiently reconverted into acetyl-CoA and butyryl-CoA, respectively, without coupling with acetone synthesis (Bao et al., 2020). The introduction of an aldehyde/alcohol dehydrogenase (encoded by adhE2) from C. acetobutylicum enabled more than $10 \mathrm{~g} / \mathrm{L}$ butanol produced from glucose (Yu et al., 2011). Recently, Zhang et al. (2018) developed a gene-editing tool applicable in C. tyrobutyricum based on its endogenous Type IB CRISPR/Cas system. They found that when the cat1 gene was replaced in-frame by adhE2, the butanol titer of the mutant reached an unprecedented $26.2 \mathrm{~g} / \mathrm{L}$. Moreover, the final concentration of by-products acetate and butyrate reached 15.2 and $2.4 \mathrm{~g} / \mathrm{L}$, respectively. High concentrations of butanol, acetate, and butyrate implied the sufficient supply of ester precursors, which 
TABLE 1 | Potential Clostridia for the production of short- and medium-chain esters.

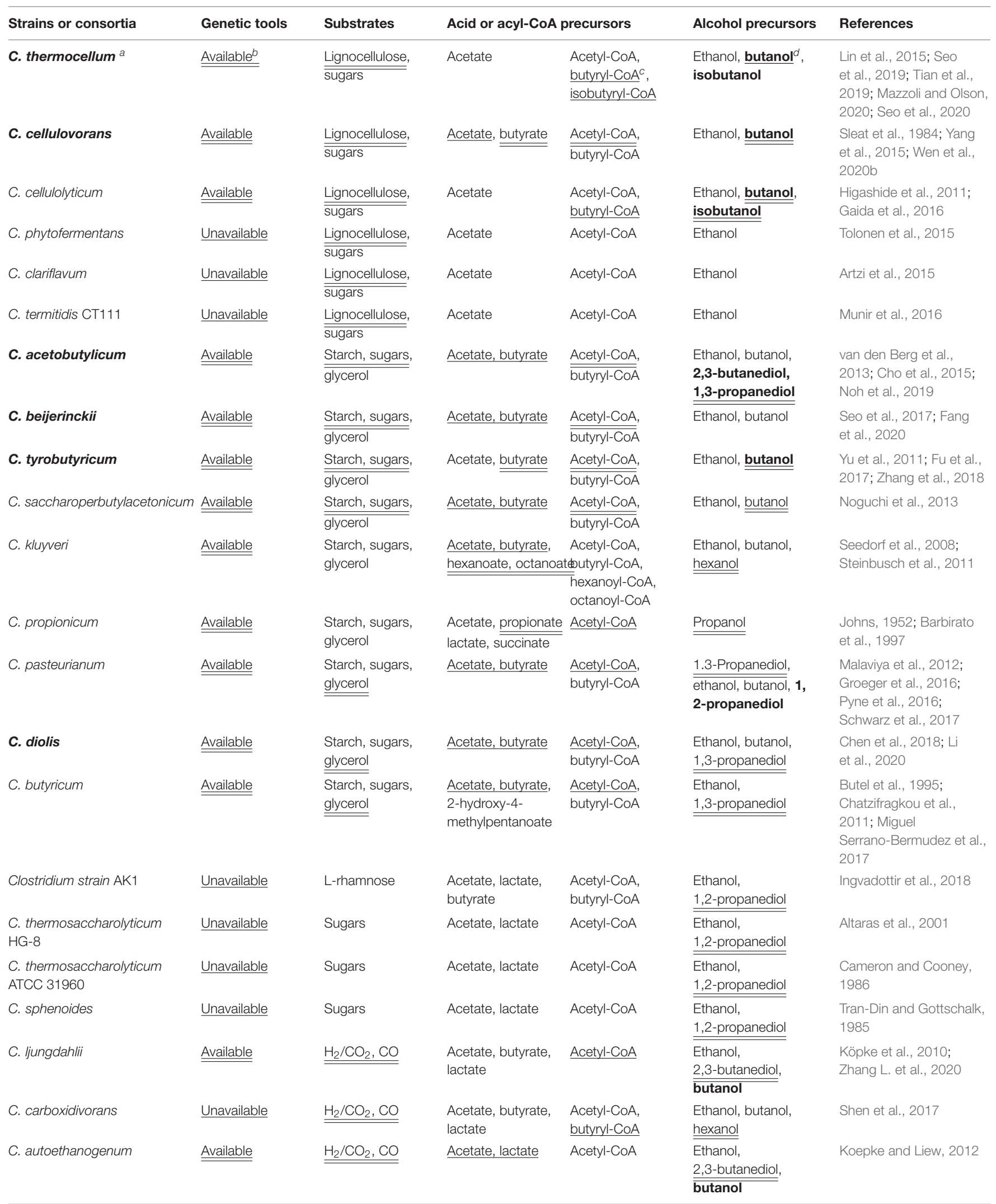


TABLE 1 | Continued

\begin{tabular}{|c|c|c|c|c|c|c|}
\hline \multirow{2}{*}{$\begin{array}{l}\text { Strains or consortia } \\
\text { C. scatologenes }\end{array}$} & \multirow{2}{*}{$\begin{array}{l}\text { Genetic tools } \\
\underline{\text { Unavailable }}\end{array}$} & \multirow{2}{*}{$\begin{array}{l}\text { Substrates } \\
\mathrm{H}_{2} / \mathrm{CO}_{2}, \mathrm{CO} \\
\end{array}$} & \multicolumn{2}{|c|}{ Acid or acyl-CoA precursors } & \multirow{2}{*}{$\begin{array}{l}\text { Alcohol precursors } \\
\text { Ethanol }\end{array}$} & \multirow{2}{*}{$\begin{array}{l}\text { References } \\
\text { Song et al., } 2014\end{array}$} \\
\hline & & & Acetate, butyrate & $\begin{array}{l}\text { Acetyl-CoA, } \\
\text { butyryl-CoA }\end{array}$ & & \\
\hline C. drakei & $\underline{\text { Unavailable }}$ & $\underline{\underline{\mathrm{H}_{2} / \mathrm{CO}_{2}, \mathrm{CO}}}$ & Acetate, butyrate & $\begin{array}{l}\text { Acetyl-CoA, } \\
\text { butyryl-CoA }\end{array}$ & Ethanol & Gossner et al., 2008 \\
\hline C. thermoaceticum & $\underline{\text { Available }}$ & $\mathrm{H}_{2} / \mathrm{CO}_{2}, \mathrm{CO}$ & Acetate & $\underline{\text { Acetyl-CoA }}$ & Ethanol & Pierce et al., 2008 \\
\hline $\begin{array}{l}\text { C. beijerinckii BGS1 and } \\
\text { C. tyrobutyricum ATCC } \\
27045\end{array}$ & Available & Starch, sugars & Acetate, butyrate & $\begin{array}{l}\text { Acetyl-CoA, } \\
\text { butyryl-CoA }\end{array}$ & Ethanol, butanol & Cui et al., 2020a \\
\hline $\begin{array}{l}\text { C. thermocellum and } \\
\text { C. saccharoperbutylacetonicum } \\
\text { strain N1-4 }\end{array}$ & $\underline{\underline{\text { Available }}}$ & $\frac{\text { Crystalline }}{\underline{\underline{\underline{\text { cellulose }}}}}$ & $\underline{\text { Acetate, butyrate }}$ & $\begin{array}{l}\text { Acetyl-CoA, } \\
\text { butyryl-CoA }\end{array}$ & Ethanol, butanol & Shunichi et al., 2011 \\
\hline $\begin{array}{l}\text { C. celevecrescens N3-2 and } \\
\text { C. acetobutylicum ATCC } 824\end{array}$ & $\underline{\text { Unavailable }}$ & Filter paper & Acetate, butyrate & $\begin{array}{l}\text { Acetyl-CoA, } \\
\text { butyryl-CoA }\end{array}$ & Ethanol, butanol & Wang et al., 2015 \\
\hline $\begin{array}{l}\text { C. cellulovorans and } \\
\text { C. beijerinckii }\end{array}$ & Available & $\underline{\underline{\text { Alkali extracted }}}$ & Acetate, butyrate & $\frac{\text { Acetyl-CoA, }}{\overline{\text { butyryl-CoA }}}$ & Ethanol, butanol & $\begin{array}{l}\text { Wen et al., 2017; Wen } \\
\text { et al., 2020a }\end{array}$ \\
\hline $\begin{array}{l}\text { C. thermocellum and } \\
\text { C. beijerinckii }\end{array}$ & Available & $\frac{\text { Alkali extracted }}{\underline{\underline{\text { corn cobs }}}}$ & $\underline{\text { Acetate, butyrate }}$ & $\begin{array}{l}\text { Acetyl-CoA, } \\
\text { butyryl-CoA }\end{array}$ & Ethanol, butanol & Wen et al., 2014 \\
\hline C. ljungdahlii and C. Kluyveri & Available & $\underline{\underline{\mathrm{H}_{2} / \mathrm{CO}_{2}, \mathrm{CO}}}$ & $\begin{array}{l}\text { Acetate, butyrate, } \\
\text { hexanoate }\end{array}$ & $\begin{array}{l}\text { Acetyl-CoA, } \\
\text { butyryl-CoA, } \\
\text { hexanoyl-CoA }\end{array}$ & Ethanol & Richter et al., 2016 \\
\hline
\end{tabular}

${ }^{a}$ Bold fonts indicate strains or consortia that have been engineered to enable ester production.

${ }^{b}$ Double underlines indicate advantages of the strain.

${ }^{c}$ Single underline indicates limitations of the strain.

${ }^{d}$ Bold fonts indicate precursors synthesized via genetic modification.

may contribute to achieve a very high titer of butyl acetate or butyl butyrate.

Similar to C. tyrobutyricum, the main product of Clostridium cellulovorans is also butyrate, while the difference is that it can directly grow on lignocellulosic biomass (Sleat et al., 1984). Interestingly, this strain harbors a complete CoAdependent butanol synthesis pathway without coupled acetone production according to the prediction of KEGG, but it can hardly produce butanol. Modular metabolic engineering has enabled the strain to produce $4.96 \mathrm{~g} / \mathrm{L}$ butanol from alkali-extracted corn cob (AECC) in $120 \mathrm{~h}$, with $4.81 \mathrm{~g} / \mathrm{L}$ butyrate and $4.14 \mathrm{~g} / \mathrm{L}$ acetate residual in broth (Wen et al., 2020b), suggesting sufficient precursors for the synthesis of butyl acetate or butyl butyrate. Recently, Fang et al. claimed that they had realized butyl acetate production in C. cellulovorans by overexpressing ATF1 and adhE1, but the detailed experimental data was not shown (Fang et al., 2020). Like C. cellulovorans, Clostridium cellulolyticum and C. thermocellum are also important cellulolytic Clostridia, and C. thermocellum has been proven to produce isobutyl acetate and isobutyl isobutyrate directly from cellulose (Seo et al., 2019; Zhang J. et al., 2020). One putative obstacle is that there are no complete pathways from acetyl-CoA to butyrate and butanol existing in C. cellulolyticum and C. thermocellum. Encouragingly, they have been successfully engineered to produce butanol (Gaida et al., 2016; Tian et al., 2019). In general, cellulolytic Clostridia offered a chance to produce esters from lignocellulose by consolidated bioprocessing.

Another reason why $C$. cellulovorans has potential as an excellent candidate host is its $\mathrm{CO}_{2}$ fixation ability (Shinohara et al., 2013), although its fixation efficiency is much lower than gas-fermenting Clostridia. Gas-fermenting Clostridia is a major type of chemoautotrophic carbon-fixing bacteria, in which Clostridium ljungdahlii, Clostridium autoethanogenum, Clostridium carboxidivorans have been adapted for ethanol and butanol production from industrial waste gas (Latif et al., 2014; Bengelsdorf and Durre, 2017). Gas-fermenting Clostridia uptake and fix $\mathrm{CO}_{2}, \mathrm{CO}$, and $\mathrm{H}_{2}$ by a Wood-Ljungdahl (WL) pathway (Fast et al., 2015). The energy metabolism and product synthesis in these strains may be completely different under different growth conditions, which result in product diversity (Bengelsdorf and Durre, 2017). Apart from acetate and ethanol, butyrate can be detected in the fermentation broth of $C$. carboxidivorans (Fernandez-Naveira et al., 2017a), Clostridium drakei (Gossner et al., 2008), Clostridium magnum (Groher and WeusterBotz, 2016), and Clostridium scatologenes (Song et al., 2014). Moreover, butanol, hexanoate, and hexanol can also be produced by C. carboxidivorans (Fernandez-Naveira et al., 2017a) and C. drakei (Fernandez-Naveira et al., 2017b). The special substrate spectrum and product (or precursor) diversity make gasfermenting Clostridia very suitable for different ester synthesis.

\section{ARTIFICIAL CLOSTRIDIAL CONSORTIA OFFER SPECIAL ADVANTAGES FOR BIO-ESTER SYNTHESIS}

Ester synthesis is a complex process involving multiple steps, such as the utilization of substrates, precursor production, lipase or AAT expression, and catalysis (Aleksander et al., 2019). A mixed-culture strategy has proven successful in complex 
biological processes (Cui et al., 2020b; Wen et al., 2020c). The members in consortia can take on different tasks and exert their unique advantages, thereby reducing the burden, expanding the spectrum of substrates, increasing product diversity, and improving the efficiency of ester synthesis. In an aforementioned study, the titer of butyl butyrate produced from coculture of $C$. beijerinckii (the butanol producer) and $C$. tyrobutyricum (the butyrate producer) is about 10-fold obtained from the C. beijerinckii monoculture, implying great potential (Cui et al., 2020a). The consortia that have been adapted for cellulosic butanol production [for example, cellulolytic C. cellulovorans and solventogenic C. beijerinckii (Wen et al., 2017)] and syngas fermentation [for example, gas-fermenting C. ljungdahlii and hexanoateproducing Clostridium kluyveri (Richter et al., 2016)] could be engineered for bio-ester production by simply introducing AATs or adding lipase.

Other Clostridia that have the potential to serve as production hosts for bio-esters but have not been discussed are summarized in Table 1. According to the table, these distinctive Clostridia and clostridial consortia endow new possibilities for bio-ester synthesis in the aspects of efficiency improvements, broad substrate spectrum, and product diversity. However, the above studies rarely involve the complicated modification of the clostridial host, including metabolic pathway reconstruction, stress resistance modification, and refined expression regulation of AATs, which implies great potential of clostridial synthetic biotechnology to improve bio-ester production.

\section{SYNTHETIC BIOLOGY WILL ACCELERATE DEVELOPMENT OF CLOSTRIDIAL ESTER CELL FACTORIES}

Although great progress has been made in bio-ester production, the clostridial potential and advantages have not been fully exploited. Synthetic biology provided many resources and methods for clostridial chassis development, which can be applied to ester production (Joseph et al., 2018; Wen et al., 2020c).

Many genetic manipulation tools such as TargeTron, allelic exchange, CRISPR/Cas system-mediated gene, and base editing tools have been developed in Clostridia (Pyne et al., 2014; McAllister and Sorg, 2019; Wen et al., 2020d). Various genetic operations such as insertion, deletion, substitution, point mutation, and regulation of target gene expression levels can be efficiently implemented in Clostridia, which laid a good foundation for metabolic engineering (Joseph et al., 2018).

\section{REFERENCES}

Aleksander, J. K. A. B., Anna, C. B. A. B., Constantinos Patinios, B. A., Youri, M. V. N. A., Mark Levisson, C., Astrid, E. M. D., et al. (2019). Microbial production of short and medium chain esters: enzymes, pathways, and applications. Biotechnol. Adv. 37:107407.
Metabolic pathway reconstruction not only increased the titer, yield, and ratio of butanol but also eliminated the byproduct acetone in some Clostridia (Cho et al., 2015; Jiang et al., 2015). In addition, it also provides a chance to synthesize some new products (Figure 1), such as long straight-chain alcohols and acids (pentanol, hexanoate, hexanol, octanoate, and octanol) or branched-chain alcohols and acids (1,2-propanediol, isopropanol, isobutanol, 2-butanol, and isopentanol) (Pyne et al., 2016; Ren et al., 2016; Bengelsdorf and Durre, 2017). These products can serve as precursors for novel esters, which may increase the diversity of ester products.

In addition, through metabolic engineering, some Clostridia has been improved in the aspects of hexose/pentose cofermentation (Gu et al., 2014; Mitchell, 2016), syngas utilization (Bengelsdorf and Durre, 2017; Valgepea et al., 2018), and efficient conversion of glycerol to butanol (Schwarz et al., 2017). These Clostridia can utilize inexpensive and renewable resources such as lignocellulosic biomass, industrial off-gas, or crude glycerol to produce bio-ester, which could further reduce bio-ester cost. However, there are still some putative drawbacks for some clostridial hosts (Moon et al., 2016), such as low recombineering efficiency, complex metabolic regulatory networks, by-products (like acetone) accumulation, and inefficient protein secretion system, which are highly dependent on synthetic biotechnology to solve.

It can be expected that with the further development of synthetic biology, bio-ester production by Clostridia will be closely combined with the novel AAT mining, rational metabolic network simulation and prediction, multi-omics analysis, and artificial consortia design. The ideas and technologies of synthetic biology will accelerate to develop Clostridia as more effective cell factories for ester production.

\section{AUTHOR CONTRIBUTIONS}

QW, XS, and ZW conceived the project and wrote the manuscript. All authors participated in the discussion, revised the manuscript, and approved the final manuscript.

\section{FUNDING}

This study was supported by the National Key Research and Development Program of China (No. 2019YFA0904900), the Tianjin Synthetic Biotechnology Innovation Capacity Improvement Project (TSBICIP-PTJS-003-04), and the grants from the National Natural Science Foundation of China (21706133, 21825804, 31670094, and 31971343).

Altaras, N. E., Etzel, M. R., and Cameron, D. C. (2001). Conversion of sugars to 1,2-propanediol by Thermoanaerobacterium thermosaccharolyticum HG-8. Biotechnol. Progress 17, 52-56. doi: 10.1021/bp000130b

Artzi, L., Morag, E., Barak, Y., Lamed, R., and Bayer, E. A. (2015). Clostridium clariflavum: key cellulosome players are revealed by proteomic analysis. Mbio 6:e0411-15. doi: 10.1128/mBio.00411-15 
Bao, T., Feng, J., Jiang, W., Fu, H., Wang, J., and Yang, S.-T. (2020). Recent advances in $\mathrm{n}$-butanol and butyrate production using engineered Clostridium tyrobutyricum. World J. Microbiol. Biotechnol. 36:138. doi: 10.1007/s11274-02002914-2

Barbirato, F., Chedaille, D., and Bories, A. (1997). Propionic acid fermentation from glycerol: comparison with conventional substrates. Appl. Microbiol. Biotechnol. 47, 441-446. doi: 10.1007/s002530050953

Bengelsdorf, F. R., and Durre, P. (2017). Gas fermentation for commodity chemicals and fuels. Microb. Biotechnol. 10, 1167-1170. doi: 10.1111/17517915.12763

Bohnenkamp, A. C., Kruis, A. J., Mars, A. E., Wijffels, R. H., van der Oost, J., Kengen, S. W. M., et al. (2020). Multilevel optimisation of anaerobic ethyl acetate production in engineered Escherichia coli. Biotechnol. Biofuels 13:65. doi: 10.1186/s13068-020-01703-1

Butel, M.-J., Rimbault, A., Khelifa, N., Campion, G., Szylit, O., and Rocchiccioli, F. (1995). Formation of 2-hydroxy-4-methylpentanoic acid from 1-leucine by Clostridium butyricum. FEMS Microbiol. Lett. 132, 171-176. doi: 10.1016/03781097(95)00306-P

Cameron, D. C., and Cooney, C. L. (1986). A novel fermentation: the production of $\mathrm{R}(-)-1,2-$ propanediol and acetol by Clostridium thermosaccharolyticum. Nat. Biotechnol. 4, 651-654. doi: 10.1038/nbt0786-651

Chatzifragkou, A., Papanikolaou, S., Dietz, D., Doulgeraki, A. I., Nychas, G. J. E., and Zeng, A. P. (2011). Production of 1,3-propanediol by Clostridium butyricum growing on biodiesel-derived crude glycerol through a non-sterilized fermentation process. Appl. Microbiol. Biotechnol. 91, 101-112.

Chen, C., Sun, C., and Wu, Y.-R. (2018). The draft genome sequence of a novel high-efficient butanol-producing bacterium clostridium diolis strain WST. Curr. Microbiol. 75, 1011-1015. doi: 10.1007/s00284-018-1481-5

Cho, C., Jang, Y.-S., Moon, H. G., Lee, J., and Lee, S. Y. (2015). Metabolic engineering of clostridia for the production of chemicals. Biofuels Bioprod. Biorefin. 9, 211-225. doi: 10.1002/bbb.1531

Cui, Y., He, J., Yang, K.-L., and Zhou, K. (2020a). Production of isopropyl and butyl esters by Clostridium mono-culture and co-culture. J. Ind. Microbiol. Biotechnol. 47, 543-550. doi: 10.1007/s10295-020-02279-3

Cui, Y., Yang, K. L., and Zhou, K. (2020b). Using co-culture to functionalize Clostridium fermentation. Trends Biotechnol. doi: 10.1016/j.tibtech.2020.11.016 [Epub ahead of print].

Cull, S. G., Holbrey, J. D., Vargas-Mora, V., Seddon, K. R., and Lye, G. J. (2003). Room-temperature ionic liquids as replacements for organic solvents in multiphase bioprocess operations. Biotechnol. Bioeng. 69, 227-233.

Fang, D., Wen, Z., Lu, M., Li, A., Ma, Y., Tao, Y., et al. (2020). Metabolic and process engineering of Clostridium beijerinckii for butyl acetate production in one step. J. Agric. Food Chem. 68, 9475-9487. doi: 10.1021/acs.jafc.0c00050

Fast, A. G., Schmidt, E. D., Jones, S. W., and Tracy, B. P. (2015). Acetogenic mixotrophy: novel options for yield improvement in biofuels and biochemicals production. Curr. Opin. Biotechnol. 33, 60-72. doi: 10.1016/j.copbio.2014.11. 014

Fernandez-Naveira, A., Abubackar, H. N., Veiga, M. C., and Kennes, C. (2017a). Production of chemicals from C1 gases (CO, CO2) by Clostridium carboxidivorans. World J. Microbiol. Biotechnol. 33:43. doi: 10.1007/s11274016-2188-z

Fernandez-Naveira, A., Veiga, M. C., and Kennes, C. (2017b). Effect of pH control on the anaerobic H-B-E fermentation of syngas in bioreactors. J. Chem. Technol. Biotechnol. 92, 1178-1185.

Fu, H., Yu, L., Lin, M., Wang, J., Xiu, Z., and Yang, S.-T. (2017). Metabolic engineering of Clostridium tyrobutyricum for enhanced butyric acid production from glucose and xylose. Metab. Eng. 40, 50-58. doi: 10.1016/j.ymben.2016.12. 014

Fujii, T., Yoshimoto, H., and Tamai, Y. (1996). Acetate ester production by Saccharomyces cerevisiae lacking the ATF1 gene encoding the alcohol acetyltransferase. J. Ferment. Bioeng. 81, 538-542. doi: 10.1016/0922-338X(96) 81476-0

Gaida, S. M., Liedtke, A., Jentges, A. H., Engels, B., and Jennewein, S. (2016). Metabolic engineering of Clostridium cellulolyticum for the production of n-butanol from crystalline cellulose. Microb Cell Fact. 15:6. doi: 10.1186/ s12934-015-0406-2

Gossner, A. S., Picardal, F., Tanner, R. S., and Drake, H. L. (2008). Carbon metabolism of the moderately acid-tolerant acetogen Clostridium drakei isolated from peat. FEMS Microbiol. Lett. 287, 236-242. doi: 10.1111/j.15746968.2008.01313.x
Groeger, C., Sabra, W., and Zeng, A.-P. (2016). Simultaneous production of 1,3propanediol and n-butanol by Clostridium pasteurianum: in situ gas stripping and cellular metabolism. Eng. Life Sci. 16, 664-674. doi: 10.1002/elsc.2016 00058

Groher, A., and Weuster-Botz, D. (2016). Comparative reaction engineering analysis of different acetogenic bacteria for gas fermentation. J. Biotechnol. 228, 82-94. doi: 10.1016/j.jbiotec.2016.04.032

Gu, Y., Jiang, Y., Yang, S., and Jiang, W. (2014). Utilization of economical substratederived carbohydrates by solventogenic clostridia: pathway dissection, regulation and engineering. Curr. Opin. Biotechnol. 29, 124-131. doi: 10.1016/j. copbio.2014.04.004

Higashide, W., Li, Y., Yang, Y., and Liao, J. C. (2011). Metabolic engineering of Clostridium cellulolyticum for production of isobutanol from cellulose. Appl. Environ. Microbiol. 77, 2727-2733. doi: 10.1128/AEM.02454-10

Horton, C. E., and Bennett, G. N. (2006). Ester production in E-coli and C-acetobutylicum. Enzyme Microb. Technol. 38, 937-943. doi: 10.1016/j. enzmictec.2005.08.025

Ingvadottir, E. M., Scully, S. M., and Orlygsson, J. (2018). Production of (S)-1,2Propanediol from L-rhamnose using the moderately thermophilic Clostridium strain AK1. Anaerobe 54, 26-30. doi: 10.1016/j.anaerobe.2018.07.003

Jermy, B. R., and Pandurangan, A. (2005). A highly efficient catalyst for the esterification of acetic acid using n-butyl alcohol. J. Mol. Catal. A Chem. 237, 146-154. doi: 10.1016/j.molcata.2005.04.034

Jiang, Y., Liu, J., Jiang, W., Yang, Y., and Yang, S. (2015). Current status and prospects of industrial bio-production of n-butanol in China. Biotechnol. Adv. 33, 1493-1501. doi: 10.1016/j.biotechadv.2014.10.007

Johns, A. T. (1952). The mechanism of propionic acid formation by Clostridium propionicum. J. Gen. Microbiol. 6, 123-127. doi: 10.1099/00221287-6-1-2-123

Joseph, R. C., Kim, N. M., and Sandoval, N. R. (2018). Recent developments of the synthetic biology toolkit for Clostridium. Front. Microbiol. 9:154. doi: 10.3389/fmicb.2018.00154

Koepke, M., and Liew, F. M. (2012). Production of Butanol from Carbon Monoxide by a Recombinant Microorganism. EP Patent Application WO 2012/053905 A1.

Köpke, M., Held, C., Hujer, S., Liesegang, H., Wiezer, A., Wollherr, A., et al. (2010). Clostridium ljungdahlii represents a microbial production platform based on syngas. Proc. Natl. Acad. Sci. U.S.A. 107:13087. doi: 10.1073/pnas.1004716107

Kruis, A. J., Gallone, B., Jonker, T., Mars, A. E., van Rijswijck, I. M. H., Wolkers-Rooijackers, J. C. M., et al. (2018a). Contribution of Eat1 and other alcohol acyltransferases to ester production in Saccharomyces cerevisiae. Front. Microbiol. 9:3202. doi: 10.3389/fmicb.2018.03202

Kruis, A. J., Levisson, M., Mars, A. E., van der Ploeg, M., Garcés Daza, F., Ellena, V., et al. (2017). Ethyl acetate production by the elusive alcohol acetyltransferase from yeast. Metab. Eng. 41, 92-101. doi: 10.1016/j.ymben.2017.03.004

Kruis, A. J., Mars, A. E., Kengen, S. W. M., Borst, J. W., van der Oost, J., and Weusthuis, R. A. (2018b). Alcohol acetyltransferase Eat1 is located in Yeast Mitochondria. Appl. Environ. Microbiol. 84:e01640-18. doi: 10.1128/ AEM.01640-18

Latif, H., Zeidan, A. A., Nielsen, A. T., and Zengler, K. (2014). Trash to treasure: production of biofuels and commodity chemicals via syngas fermenting microorganisms. Curr. Opin. Biotechnol. 27, 79-87. doi: 10.1016/j.copbio.2013. 12.001

Lee, S. Y., Park, J. H., Jang, S. H., Nielsen, L. K., Kim, J., and Jung, K. S. (2008). Fermentative butanol production by clostridia. Biotechnol. Bioeng. 101, 209-228. doi: 10.1002/bit.22003

Li, A., Wen, Z., Fang, D., Lu, M., Ma, Y., Xie, Q., et al. (2020). Developing Clostridium diolis as a biorefinery chassis by genetic manipulation. Bioresour. Technol. 305:123066. doi: 10.1016/j.biortech.2020.123066

Lilly, M., Bauer, F. F., Lambrechts, M. G., Swiegers, J. H., Cozzolino, D., and Pretorius, I. S. (2006). The effect of increased yeast alcohol acetyltransferase and esterase activity on the flavour profiles of wine and distillates. Yeast 23, 641-659. doi: 10.1002/yea.1382

Lin, P. P., Mi, L., Morioka, A. H., Yoshino, K. M., Konishi, S., Xu, S. C., et al. (2015). Consolidated bioprocessing of cellulose to isobutanol using Clostridium thermocellum. Metab. Eng. 31, 44-52. doi: 10.1016/j.ymben.2015.07.001

Löbs, A. K., Engel, R., Schwartz, C., Flores, A., and Wheeldon, I. (2017). CRISPR-Cas9-enabled genetic disruptions for understanding ethanol and ethyl acetate biosynthesis in Kluyveromyces marxianus. Biotechnol. Biofuels 10:164.

Malaviya, A., Jang, Y.-S., and Lee, S. Y. (2012). Continuous butanol production with reduced byproducts formation from glycerol by a hyper producing mutant 
of Clostridium pasteurianum. Appl. Microbiol. Biotechnol. 93, 1485-1494. doi: 10.1007/s00253-011-3629-0

Mazzoli, R., and Olson, D. G. (2020). Clostridium thermocellum: a microbial platform for high-value chemical production from lignocellulose. Adv. Appl. Microbiol. 113, 111-161. doi: 10.1016/bs.aambs.2020.07.004

McAllister, K. N., and Sorg, J. A. (2019). CRISPR genome editing systems in the genus Clostridium: a timely advancement. J. Bacteriol. 201:00219-19. doi: 10.1128/jb.00219-19

Miguel Serrano-Bermudez, L., Fernando Gonzalez Barrios, A., Maranas, C. D., and Montoya, D. (2017). Clostridium butyricum maximizes growth while minimizing enzyme usage and ATP production: metabolic flux distribution of a strain cultured in glycerol. BMC Syst. Biol. 11:58. doi: 10.1186/s12918-0170434-0

Mitchell, W. J. (2016). Sugar uptake by the solventogenic clostridia. World J. Microbiol. Biotechnol. 32:32. doi: 10.1007/s11274-015-1981-4

Moon, H. G., Jang, Y.-S., Cho, C., Lee, J., Binkley, R., and Lee, S. Y. (2016). One hundred years of clostridial butanol fermentation. FEMS Microbiol. Lett. 363:fnw001. doi: 10.1093/femsle/fnw001

Mukdsi, M. C. A., Medina, R. B., Alvarez, M. D. F., and Gonzalez, S. N. (2009). Ester synthesis by lactic acid bacteria isolated from goat's and ewe's milk and cheeses. Food Chem. 117, 241-247.

Munir, R. I., Spicer, V., Krokhin, O. V., Shamshurin, D., Zhang, X. L., Taillefer, M., et al. (2016). Transcriptomic and proteomic analyses of core metabolism in Clostridium termitidis CT1112 during growth on alpha-cellulose, xylan, cellobiose and xylose. BMC Microbiol. 16:91. doi: 10.1186/s12866-016-0711-x

Noguchi, T., Tashiro, Y., Yoshida, T., Zheng, J., Sakai, K., and Sonomoto, K. (2013). Efficient butanol production without carbon catabolite repression from mixed sugars with Clostridium saccharoperbutylacetonicum N1-4. J. Biosci. Bioeng. 116, 716-721. doi: 10.1016/j.jbiosc.2013.05.030

Noh, H. J., Lee, S. Y., and Jang, Y.-S. (2019). Microbial production of butyl butyrate, a flavor and fragrance compound. Appl. Microbiol. Biotechnol. 103, 2079-2086. doi: $10.1007 /$ s00253-018-09603-Z

Noh, H. J., Woo, J. E., Lee, S. Y., and Jang, Y.-S. (2018). Metabolic engineering of Clostridium acetobutylicum for the production of butyl butyrate. Appl. Microbiol. Biotechnol. 102, 8319-8327. doi: 10.1007/s00253-018-9267-z

Pierce, E., Xie, G., Barabote, R. D., Saunders, E., Han, C. S., Detter, J. C., et al. (2008). The complete genome sequence of Moorella thermoacetica (f. Clostridium thermoaceticum). Environ. Microbiol. 10, 2550-2573.

Pyne, M. E., Bruder, M., Moo-Young, M., Chung, D. A., and Chou, C. P. (2014). Technical guide for genetic advancement of underdeveloped and intractable Clostridium. Biotechnol. Adv. 32, 623-641. doi: 10.1016/j.biotechadv.2014.04. 003

Pyne, M. E., Sokolenko, S., Liu, X., Srirangan, K., Bruder, M. R., Aucoin, M. G., et al. (2016). Disruption of the reductive 1,3-propanediol pathway triggers production of 1,2-propanediol for sustained glycerol fermentation by Clostridium pasteurianum. Appl. Environ. Microbiol. 82, 5375-5388. doi: 10. 1128/aem.01354-16

Ren, C., Wen, Z., Xu, Y., Jiang, W., and Gu, Y. (2016). Clostridia: a flexible microbial platform for the production of alcohols. Curr. Opin. Chem. Biol. 35, 65-72. doi: 10.1016/j.cbpa.2016.08.024

Richter, H., Molitor, B., Diender, M., Sousa, D. Z., and Angenent, L. T. (2016). A narrow ph range supports butanol, hexanol, and octanol production from syngas in a continuous co-culture of Clostridium ljungdahlii and clostridium kluyveri with in-line product extraction. Front. Microbiol. 7:1773. doi: 10.3389/ fmicb.2016.01773

Rodriguez, G. M., Tashiro, Y., and Atsumi, S. (2014). Expanding ester biosynthesis in Escherichia coli. Nat. Chem. Biol. 10:259. doi: 10.1038/nchembio.1476

Saerens, S. M., Verstrepen, K. J., Van Laere, S. D., Voet, A. R., Van Dijck, P., Delvaux, F. R., et al. (2006). The Saccharomyces cerevisiae EHT1 and EEB1 genes encode novel enzymes with medium-chain fatty acid ethyl ester synthesis and hydrolysis capacity. J. Biol. Chem. 281, 4446-4456. doi: 10.1074/ jbc.M512028200

Schwarz, K. M., Grosse-Honebrink, A., Derecka, K., Rotta, C., Zhang, Y., and Minton, N. P. (2017). Towards improved butanol production through targeted genetic modification of Clostridium pasteurianum. Metab. Eng. 40, 124-137. doi: 10.1016/j.ymben.2017.01.009
Seedorf, H., Fricke, W. F., Veith, B., Bruggemann, H., Liesegang, H., Strittimatter, A., et al. (2008). The genome of Clostridium kluyveri, a strict anaerobe with unique metabolic features. Proc. Natl. Acad. Sci. U.S.A. 105, 2128-2133.

Seo, H., Lee, J.-W., Garcia, S., and Trinh, C. T. (2019). Single mutation at a highly conserved region of chloramphenicol acetyltransferase enables isobutyl acetate production directly from cellulose by Clostridium thermocellum at elevated temperatures. Biotechnol. Biofuels 12:245. doi: 10.1186/s13068-019-1583-8

Seo, H., Nicely, P. N., and Trinh, C. T. (2020). Endogenous carbohydrate esterases of Clostridium thermocellum are identified and disrupted for enhanced isobutyl acetate production from cellulose. Biotechnol. Bioeng. 117, 2223-2236. doi: 10.1002/bit.27360

Seo, S.-O., Wang, Y., Lu, T., Jin, Y.-S., and Blaschek, H. P. (2017). Characterization of a Clostridium beijerinckii spo0A mutant and its application for butyl butyrate production. Biotechnol. Bioeng. 114, 106-112. doi: 10.1002/bit.26057

Shen, S., Gu, Y., Chai, C., Jiang, W., Zhuang, Y., and Wang, Y. (2017). Enhanced alcohol titre and ratio in carbon monoxide-rich off-gas fermentation of Clostridium carboxidivorans through combination of trace metals optimization with variable-temperature cultivation. Bioresour. Technol. 239, 236-243. doi: 10.1016/j.biortech.2017.04.099

Shinohara, M., Sakuragi, H., Morisaka, H., Miyake, H., Tamaru, Y., Fukusaki, E., et al. (2013). Fixation of $\mathrm{CO} 2$ in Clostridium cellulovorans analyzed by 13C-isotopomer-based target metabolomics. AMB Express 3:61.

Shunichi, N., Keiji, K., Toshimori, K., and Atsumi, N. (2011). Butanol production from crystalline cellulose by cocultured Clostridium thermocellum and Clostridium saccharoperbutylacetonicum N1-4. Appl. Environ. Microbiol. 77, 6470-6475.

Sleat, R., Mah, R. A., and Robinson, R. (1984). Isolation and characterization of an anaerobic, cellulolytic bacterium, Clostridium cellulovorans sp. nov. Appl. Environ. Microbiol. 48, 88-93.

Song, Y., Jeong, Y., Shin, H. S., and Cho, B. K. (2014). Draft genome sequence of Clostridium scatologenes ATCC 25775, a chemolithoautotrophic acetogenic bacterium producing 3-methylindole and 4-methylphenol. Genome Announc. 2:e00459-14. doi: 10.1128/genomeA.00459-14

Steinbusch, K. J. J., Hamelers, H. V. M., Plugge, C. M., and Buisman, C. J. N. (2011). Biological formation of caproate and caprylate from acetate: fuel and chemical production from low grade biomass. Energy Environ. Sci. 4, 216-224.

Stergiou, P. Y., Foukis, A., Filippou, M., Koukouritaki, M., Parapouli, M., Theodorou, L. G., et al. (2013). Advances in lipase-catalyzed esterification reactions. Biotechnol. Adv. 31, 1846-1859. doi: 10.1016/j.biotechadv.2013.08. 006

Tankov, I., Mitkova, M., Nikolova, R., Veli, A., and Stratiev, D. (2017). n-Butyl acetate synthesis in the presence of pyridinium-based acidic ionic liquids: influence of the anion nature. Catal. Lett. 147, 2279-2289. doi: 10.1007/s10562017-2135-0

Tian, L., Conway, P. M., Cervenka, N. D., Cui, J., Maloney, M., Olson, D. G., et al. (2019). Metabolic engineering of Clostridium thermocellum for n-butanol production from cellulose. Biotechnol. Biofuels 12:186. doi: 10.1186/s13068019-1524-6

Tolonen, A. C., Zuroff, T. R., Ramya, M., Boutard, M., Cerisy, T., and Curtis, W. R. (2015). Physiology, genomics, and pathway engineering of an ethanoltolerant strain of Clostridium phytofermentans. Appl. Environ. Microbiol. 81, 5440-5448. doi: 10.1128/aem.00619-15

Tracy, B. P., Jones, S. W., Fast, A. G., Indurthi, D. C., and Papoutsakis, E. T. (2012). Clostridia: the importance of their exceptional substrate and metabolite diversity for biofuel and biorefinery applications. Curr. Opin. Biotechnol. 23, 364-381. doi: 10.1016/j.copbio.2011.10.008

Tran-Din, K., and Gottschalk, G. (1985). Formation of d(-)-1,2-propanediol and $\mathrm{d}(-)$-lactate from glucose by Clostridium sphenoides under phosphate limitation. Arch. Microbiol. 142, 87-92. doi: 10.1007/BF00409243

Valgepea, K., Lemgruber, R. D. S. P., Abdalla, T., Binos, S., Takemori, N., Takemori, A., et al. (2018). H-2 drives metabolic rearrangements in gas-fermenting Clostridium autoethanogenum. Biotechnol. Biofuels 11:55. doi: 10.1186/s13068018-1052-9

van den Berg, C., Heeres, A. S., van der Wielen, L. A. M., and Straathof, A. J. J. (2013). Simultaneous clostridial fermentation, lipase-catalyzed esterification, and ester extraction to enrich diesel with butyl butyrate. Biotechnol. Bioeng. 110, 137-142. doi: 10.1002/bit.24618 
Wang, Z., Cao, G., Zheng, J., Fu, D., Song, J., Zhang, J., et al. (2015). Developing a mesophilic co-culture for direct conversion of cellulose to butanol in consolidated bioprocess. Biotechnol. Biofuels 8, 1-9.

Wen, Z., Ledesma-Amaro, R., Lu, M., Jiang, Y., Gao, S., Jin, M., et al. (2020a). Combined evolutionary engineering and genetic manipulation improve low $\mathrm{pH}$ tolerance and butanol production in a synthetic microbialClostridiumcommunity. Biotechnol. Bioeng. 117, 2008-2022. doi: 10.1002/bit.27333

Wen, Z., Ledesma-Amaro, R., Lu, M., Jin, M., and Yang, S. (2020b). Metabolic engineering of Clostridium cellulovorans to improve butanol production by consolidated bioprocessing. ACS Synth. Biol. 9:304. doi: 10.1021/acssynbio. 9 b00331

Wen, Z., Li, Q., Liu, J., Jin, M., and Yang, S. (2020c). Consolidated bioprocessing for butanol production of cellulolytic Clostridia: development and optimization. Microb. Biotechnol. 13, 410-422. doi: 10.1111/1751-7915.13478

Wen, Z., Lu, M., Ledesma-Amaro, R., Li, Q., Jin, M., and Yang, S. (2020d). TargeTron technology applicable in solventogenic Clostridia: revisiting 12 Years' advances. Biotechnol. J. 15:e1900284. doi: 10.1002/biot.201900284

Wen, Z., Minton, N. P., Zhang, Y., Li, Q., Liu, J., Jiang, Y., et al. (2017). Enhanced solvent production by metabolic engineering of a twin-clostridial consortium. Metab. Eng. 39, 38-48. doi: 10.1016/j.ymben.2016.10.013

Wen, Z., Wu, M., Lin, Y., Yang, L., Lin, J., and Cen, P. (2014). A novel strategy for sequential co-culture of Clostridium thermocellum and Clostridium beijerinckii to produce solvents from alkali extracted corn cobs. Process Biochem. 49, 1941-1949. doi: 10.1016/j.procbio.2014.07.009

Wiegel, J., Tanner, R., and Rainey, F. A. (2006). "An introduction to the family clostridiaceae," in The Prokaryotes. (eds) M. Dworkin, S. Falkow, E. Rosenberg, K. H. Schleifer, and E. Stackebrandt (New York, NY: Springer)

Xin, F., Basu, A., Yang, K.-L., and He, J. (2016). Strategies for production of butanol and butyl-butyrate through lipase-catalyzed esterification. Bioresour. Technol. 202, 214-219. doi: 10.1016/j.biortech.2015.11.068

Yang, X., Xu, M., and Yang, S. T. (2015). Metabolic and process engineering of Clostridium cellulovorans for biofuel production from cellulose. Metab. Eng. 32, 39-48. doi: 10.1016/j.ymben.2015.09.001

Yi, Z., Jin, Y., Xiao, Y., Chen, L., Tan, L., Du, A., et al. (2019). Unraveling the contribution of high temperature stage to Jiang-Flavor Daqu, a liquor starter for production of Chinese Jiang-flavor Baijiu, with special reference to metatranscriptomics. Front. Microbiol. 10:472. doi: 10.3389/fmicb.2019.00472

Yoshimoto, H., Fujiwara, D., Momma, T., Ito, C., Sone, H., Kaneko, Y., et al. (1998). Characterization of the ATF1 and Lg-ATF1 genes encoding alcohol acetyltransferases in the bottom fermenting yeast Saccharomyces pastorianus. J. Ferment. Bioeng. 86, 15-20. doi: 10.1016/S0922-338X(98)80027-5

Yu, M., Zhang, Y., Tang, I. C., and Yang, S.-T. (2011). Metabolic engineering of Clostridium tyrobutyricum for n-butanol production. Metab. Eng. 13, 373-382. doi: 10.1016/j.ymben.2011.04.002

Zhang, J., Hong, W., Guo, L., Wang, Y., and Wang, Y. (2020). Enhancing plasmid transformation efficiency and enabling CRISPR-Cas9/Cpfl-based genome editing in Clostridium tyrobutyricum. Biotechnol. Bioeng. 117, 2911-2917. doi: 10.1002/BIT.27435

Zhang, J., Zong, W., Hong, W., Zhang, Z.-T., and Wang, Y. (2018). Exploiting endogenous CRISPR-Cas system for multiplex genome editing in Clostridium tyrobutyricum and engineer the strain for high-level butanol production. Metab. Eng. 47, 49-59. doi: 10.1016/j.ymben.2018.03.007

Zhang, L., Zhao, R., Jia, D., Jiang, W., and Gu, Y. (2020). Engineering Clostridium ljungdahlii as the gas-fermenting cell factory for the production of biofuels and biochemicals. Curr. Opin. Chem. Biol. 59, 54-61. doi: 10.1016/j.cbpa.2020.04. 010

Zhang, Z.-T., Taylor, S., and Wang, Y. (2017). In situ esterification and extractive fermentation for butyl butyrate production with Clostridium tyrobutyricum. Biotechnol. Bioeng. 114, 1428-1437. doi: 10.1002/bit.26289

Conflict of Interest: The authors declare that the research was conducted in the absence of any commercial or financial relationships that could be construed as a potential conflict of interest.

Copyright (c) 2021 Wang, Al Makishah, Li, Li, Liu, Sun, Wen and Yang. This is an open-access article distributed under the terms of the Creative Commons Attribution License (CC BY). The use, distribution or reproduction in other forums is permitted, provided the original author(s) and the copyright owner(s) are credited and that the original publication in this journal is cited, in accordance with accepted academic practice. No use, distribution or reproduction is permitted which does not comply with these terms. 